\title{
Therapeutic Effects of Integrated Traditional Chinese Medicine and Western Medicine in Treating Severe Acute Respiratory Syndrome
}

\author{
WANG Rong-bing (王融冰), LIU Jun-min (刘军民), JIANG Yu-yong(江宇泳), WU Yun-zhong(吴云忠), \\ WANG Xiao-jing(王晓静), CHI Pin-pin(池频频), SUN Feng-xia(孙风霞), and GAO Lian-yin (高连印)
}

\begin{abstract}
Objective: To improve the effects of treatment of severe acute respiratory syndrome (SARS) and to explore the clinical significance of integrated traditional Chinese medicine and western medicine (ICWM) in the treatment of SARS and its influence on the chief indexes in the process of the disease. Methods: The clinical study involving observation of 135 patients of SARS was conducted in the randomized, synchronously controlled and open way. The patients were divided into two groups, 68 in the ICWM group and 67 in the control group, all of whom were treated with the same basic treatment of western medicine, but to the ICWM group, Chinese drugs for clearing Heat, detoxifying and removing Dampness were given additionally. The comprehensive effect on relieving fever, cell-mediated immunity, pulmonary inflammation and secondary infection was compared between the two groups. Results: The therapeutic effect in the ICWM group was better than that in the control group in such aspects as steadily lowering body temperature, alleviating general symptoms, accelerating the absorption of pulmonary infiltration and easing cellular immunity suppression. Conclusion: The therapeutic effect of ICWM is better in treating SARS than that of western medicine alone.
\end{abstract}

KEY WORDS integrated traditional Chinese and western medicinal therapy, severe acute respiratory syndrome, synchronous controlled study

Severe acute respiratory syndrome (SARS) is an acute infectious disease caused by a new kind of coronavirus, and as it is strongly infective, highly mortal and liable to affect human beings, it severely threatens human health, influences their daily life and productive activity. Now, the microbiological characteristics of SARS related virus and its infection mechanism are being deeply studied but there has been no specific treatment for SARS found so far. In order to elevate the therapeutic effects, lighten patients' symptoms, improve the pulmonary inflammation and cellular immune inhibition that occurred in the course of the illness, a clinical study of the treatment of 68 SARS patients with integrated traditional Chinese and western medicine (ICWM), which was controlled with 67 patients treated with western medicine alone, was carried out. The results are reported as follows.

\section{METHODS}

\section{Standard for Diagnosing and Typing}

The Standard for Diagnosis, Typing and Discharging of SARS Patients promulgated in May 3rd 2003 by the Ministry of Health was adopted.

Patients younger than 18 years or older than 65 years and complicated with severe systemic diseases or women in pregnant or lactation stage were all excluded from this study.

\section{General Materials}

The 135 patients enrolled were inpatients of the authors' hospital, hospitalized between April 10th and May 26th, 2003, with their diagnosis confirmed. The patients were divided according to the sequence of enrolling into two groups. The 68 patients in the treated group were 27 males and 41 females; aged from 18 to 56 years, $35.5 \pm 10.8$ years on average; their course of disease $6.41 \pm$ 4.14 days; 36 of them were of the general type and 32 of the severe type. The 67 patients in the control group were 23 males and 44 females; aged from 20 to 65 years, $38.7 \pm 11.4$ years on average; their course of disease $7.54 \pm 4.69$ days; 39 of them were of the general type and 28 of the severe type. The two groups were not different in sex, age, course of disease, clinical typing as well as their lymphocyte count and X-ray chest manifestation, and thus they are comparable.

\section{Treatment}

The basic treatment recommended by the group of SARS Specialists from the Command Cen-

Beijing Ditan Hospital, Beijing (100011)

Correspondence to: Dr. WANG Rong-bing

Tel/Fax: 010-64211031-2328

E-mail: jyy70@126.com 
ter of the Ministry of Health was applied to both groups, which includes intravenous injection of methylprednisolone $80-160 \mathrm{mg}$ and Ribavirin 0 . $75-1.5 \mathrm{~g}$ per day for 2 weeks; Azithromycin 0.5 $\mathrm{g}$ per day for 5 days; Levofloxacin $0.4 \mathrm{~g}$ per day for 7 days; Thymus peptide $100-200 \mathrm{mg}$ per day with the course of treatment and the general supportive therapy unlimited. All the patients received oxygen inhalation by nasal tube, and mechanical ventilation was used when necessary.

Based on the above-mentioned basic treatment, Chinese drug therapy was given additionally to the treated group following the therapeutic program recommended by the Specialists Group of State TCM Administration. For patients in the developing stage, Guoyao No.2 (国药 2 号), consisting mainly of gypsum, bitter apricot seed, scutellaria root, atractylodes rhizome, ternate pinellia rhizome, purple gromwell, etc. was given; for patients in the critical stage, Guoyao No.3 (国药 3 号), consisting mainly of buffalo horn, raw rehmannia root, figwort root, Honeysuckle flower, etc. was given; and for those in the recovery stage or with the course of disease over 3 weeks, Guoyao No.4 (国药 4 号), consisting of glehnia root, dwarf lilyturf root, red sage root, red peony root, American ginseng, etc. was given. All the recipes were given one dose a day, made into decoction of $200 \mathrm{ml}$, and taken $100 \mathrm{ml}$ in the morning and evening respectively. The course of TCM treatment to all the patients was over 2 weeks.

\section{Items and Methods of Observation}

1. Changes of symptoms and signs of all patients in the therapeutic course were recorded.

2. Physical and chemical examinations were carried out before and after treatment to observe the changes of body temperature, blood routine, sero-zymogram, T-lymphocyte and its subsets, and $\mathrm{X}$-ray chest figure.

3. In part of the patients, pharyngeal gargle and urine were taken to detect the SARS-associated coronavirus (SARS-CoV) RNA by PCR and their blood level of anti-SARS-CoV IgG was detected by ELISA. These tests were performed by the National Center for Diseases Control (CDC) or the Beijing CDC.

4. Occurrence of complications, especially secondary infection, were observed in patients whose course of illness was over 4 weeks.

\section{Statistical Analysis}

$t$-test, $\chi^{2}$ test and rank-sum test were adopted with SPSS 10.0 software.

\section{RESULTS}

\section{Clinical Therapeutic Effects}

According to literature ${ }^{(1)}$, a patient who had his/her body temperature lowered to normal range for over 7 days, symptoms of respiratory tract obviously improved and the lung lesion in X-ray chest film significantly absorbed was regard as the standard for discharged from hospital, otherwise, he or she was considered as un-cured.

\section{Comparison of Etiological Parameters be- tween the Two Groups}

Shown in Table 1. Results showed that the positive rate of SARS-CoV RNA in pharyngeal gargle tested during hospitalization (T1), and that in urine tested 20 days after hospitalization (T2), as well as the blood anti-SARS-CoV-IgG (T3) detection were similar in the two groups, showing insignificant difference between them ( all $P>0.05)$.

Table 1. Comparison of Etiological Parameters between the Two Groups

\begin{tabular}{|c|c|c|c|c|c|c|}
\hline \multirow[b]{2}{*}{ Gruop } & \multicolumn{2}{|r|}{$\mathrm{T} 1$} & \multicolumn{2}{|r|}{$\mathrm{T} 2$} & \multicolumn{2}{|r|}{ T3 } \\
\hline & $n$ & $\begin{array}{l}\text { Positive } \\
\text { Rate }(\%)\end{array}$ & $n$ & $\begin{array}{c}\text { Positive } \\
\text { Rate(\%) }\end{array}$ & $n$ & $\begin{array}{c}\text { Positive } \\
\text { Rate }(\%)\end{array}$ \\
\hline Treated & 29 & 62.07 & 53 & 73.58 & 23 & 4.35 \\
\hline Control & 25 & 72.00 & 17 & 76.47 & 8 & 0 \\
\hline
\end{tabular}

\section{Comparison of Defervescent Effect between the Two Groups}

As shown in Figure 1, the body temperature of patients in the two groups before treatment was not different significantly. Two and three days after admission, the body temperature of patients in the control group was significantly higher than that in the treated group $(P<0.01)$, and it was only in the time of the 4 th day to the 14 th day after admission that the body temperature in the two

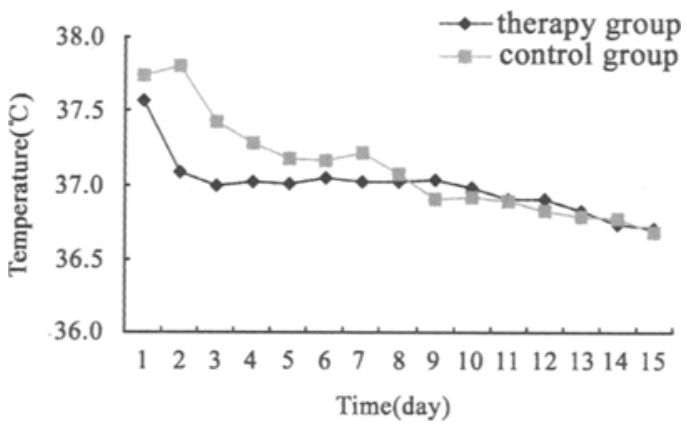

Fig. 1 Body Temperature of the Patients in the Two Groups 
groups came to be equal finally, suggesting that the defervescent effect of ICWM treatment was on an even keel.

\section{Effect on Cellular Immune Function}

As shown in Table 2, the lymphocyte count and lymphocyte subsets CD3 and CD4 in most patients of both groups before treatment were low, especially in the treated group, but the inter-group comparison showed no significant difference (the non-normally distributed material was analyzed with rank-sum test, $P<0.05)$. The condition of the ASRS patients with continuous low lymphocytes count was always severe. The three parameters all raised in the recovery stage, and the increment of CD3, CD4 and CD8 was all higher in the treated group than that in the control group respectively, showing significant statistical difference $(P<0.05$ or $P<0.01)$, suggesting that the ICWM treatment has rather better effects in protecting cellular immune and promoting recovery of immunity.

\section{Effect on Absorption of Inflammation in the Lung}

As shown in Table 3, before treatment, inflammation of various degrees appeared in all the patients' lung. After being treated for 3 weeks, by Xray examination, it was found that the inflammation was improved to some extent or basically absorbed, and the number of patients with their lesion completely absorbed in the treated group was higher than that in the control group $(P<0.05)$, but the absorption time between the two group was not different significantly.

\section{Comparison of the Dose and Time of Methyl- prednisolone Used between the Two Groups}

As shown in Table 4, the total dosage, time and the daily average dose in individual patients of methylprednisolone used in the treated group were lower than those in the control group, but showed no statistical inter-group significant difference (by rank-sum analysis, $P>0.05$ )

Table 4. Comparison of the Dose and Time of Using Methylprednisolone between Groups

\begin{tabular}{lcccr}
\hline & \multicolumn{3}{c}{ Methylprenisolone Used } \\
\cline { 2 - 4 } Gruop & $n$ & $\begin{array}{c}\text { Total Dosa } \\
(\bar{x} \pm s, \mathrm{mg})\end{array}$ & $\begin{array}{c}\text { Time } \\
(\bar{x} \pm s, \text { days })\end{array}$ & $\begin{array}{c}\text { Av. Daily Dose } \\
(\mathrm{mg} / \text { Patient })\end{array}$ \\
\hline Treated & 51 & $1466.9 \pm 625.0$ & $16.0 \pm 4.7$ & $91.8 \pm 30.8$ \\
Control & 48 & $1823.5 \pm 836.6$ & $17.8 \pm 7.2$ & $102.4 \pm 42.0$ \\
\hline
\end{tabular}

\section{Effect on Secondary Infection and Complications}

The number of patients suffering from secondary bacterial or fungal infections, or complicated with digestive tract bleeding in the treated group were $5(7.4 \%), 2(2.9 \%)$ and zero respectively, which were less than those in the control group: 9 $(13.4 \%), 5(7.5 \%)$ and $4(6.0 \%)$ respectively, but the difference between the two groups showed no statistical significance (all $P>0.05$ ). Since decreasing and controlling secondary infection was of vital importance to lowering of mortality, it may be something that explains the better therapeutic effect obtained by ICWM.

Among the 68 patients in the treated group, $66(97.1 \%)$ were discharged and $2(2.9 \%)$ uncured, while in the 67 patients in the control group, $58(86.6 \%)$ cured and $9(13.4 \%)$ uncured, comparison between the two groups showed significant difference $(P<0.05)$.

Table 2. Effects on Cellular Immune Function $(\vec{x} \pm s)$

\begin{tabular}{llcrll}
\hline Group & & Lymphocyte $\left(\times 10^{9} / \mathrm{L}\right)$ & CD3 $($ cell $/ \mu 1)$ & CD4 (cell $/ \mu \mathrm{l})$ & CD8(cell $/ \mu \mathrm{l})$ \\
\hline Treated & BT & $0.67 \pm 0.19(40)$ & $451.68 \pm 218.39(63)$ & $256.97 \pm 155.12(66)$ & $163.75 \pm 71.72(55)$ \\
& AT & $1.84 \pm 0.12(40)$ & $1182.48 \pm 67.24(63)^{*}$ & $695.21 \pm 46.33(66)^{*}$ & $421.65 \pm 27.30(55)^{* *}$ \\
Control & BT & $0.65 \pm 0.20(42)$ & $491.39 \pm 222.35(56)$ & $283.72 \pm 157.77(60)$ & $188.52 \pm 77.44(48)$ \\
& AT & $1.54 \pm 0.14(43)$ & $1034.38 \pm 70.94(56)$ & $570.29 \pm 40.36(60)$ & $389.81 \pm 36.40(48)$ \\
\hline
\end{tabular}

Notes: BT: before treatment, AT: after treatment; ${ }^{*} P<0.05,{ }^{* *} P<0.01$, compared with the same group BT; the number in the bracket is the case number

Table 3. Effect on Inflammatory Lesion in the Lung

\begin{tabular}{|c|c|c|c|c|c|c|}
\hline \multirow[b]{2}{*}{ Group } & \multirow[b]{2}{*}{ Cases } & \multicolumn{2}{|c|}{ Type of Lesion (Cases) } & \multicolumn{2}{|c|}{ Outcome of Lesion (Cases) } & \multirow[b]{2}{*}{$\begin{array}{l}\text { Absorption Time } \\
\text { (days, } \bar{x} \pm s)\end{array}$} \\
\hline & & Single lobar & Multi-lobar & $\begin{array}{l}\text { Absorbed } \\
\text { Completely }\end{array}$ & $\begin{array}{l}\text { Absorbed } \\
\text { Partially }\end{array}$ & \\
\hline Treated & 68 & 12 & 56 & 48 & 20 & $18.58 \pm 1.00$ \\
\hline Control & 67 & 13 & 52 & 33 & 34 & $17.00 \pm 6.08$ \\
\hline
\end{tabular}




\section{DISCUSSION}

The ICWM therapeutic mode is something special in Chinese clinical medicine, and ICWM treatment has won certain effect in treating SARS. At the present there is no specific treatment directly against SARS virus, and the mechanism of SARS is still unclear. Western medicine is used as symptomatic and supporting therapy, taking inhibition of the super-strong immune reaction and controlling the complication as its aim of treatment, while Chinese medicine is used, starting from Syndrome Differentiation, combining the old and new experience in treating febrile diseases, to form various therapeutic principles suitable to different phases, with clearing Heat, removing Dampness and benefiting Fei (肺) as the mainstay of treatment throughout the whole course. ICWM treatment could, getting TCM and WM complementing each other, reduce the adverse effect of drugs and enhance the integral therapeutic effect.

Fever is a common symptom revealed in SARS patients. Though a self-protection mechanism of the body, it could induce serious accompanying pathological affection. In this study, it was shown that fever was relieved rapidly in all the patients in both groups due to the rapid defervescent effect of cortical steroid, which covered the fever abating effect of Chinese drugs. However, the mitigatively deprived curve of body temperature in the treated group showed that medication of Chinese drugs could abate the body temperature and improve systemic symptoms on an even keel through some mechanisms in inhibiting virus induced immune injury to relieve the inflammatory reaction. Clinical observation also indicated that Chinese drug predominated therapy is safe and effective for treatment of patients with no serious toxic symptoms or unsuitable to be treated by cortical steroid.

Obvious deprivation of lymphocytes, which indicates the damage of immune mechanism of the organism, has not only significance in diagnosis, but also in prognosis of the disease. This study shows that ICWM can reduce the extent of lymphopenia effectively and improve the restoring of lymphocytes in the recovery stage, indicating that Chinese drugs can protect cellular immunity or antagonize the inhibition of cortical steroid on cellular immunity. Although the clear mechanism is still awaiting for further study, the results of this study has already illustrated the effect of Chinese medical concept of regulating body immunity through adjusting Yin-Yang, maintaining stability of inner environment in human body, supporting the healthy energy and dispelling evil-pathogens and improving stress response.

The pathogenesis of SARS is still under research. It has been confirmed that one of the causes of SARS is the immuno-pathological damage, and with the lung as the target organ, its pathological basis is the endothelial injury of pulmonary capillaries, which further induced the inflammatory change of alveoli, hyaline membrane formation, interstitial inflammation and consolidation of the lung. Therefore to hold back the developing of pulmonary lesions and promote the absorption of inflammation are important therapeutic ways against SARS. The better therapeutic effects in repairing pulmonary injury and inflammation absorption shown in the treated group in this study may be related with the effects of Chinese drugs in lightening immuno-pathological damage, disintegrating toxic metabolites, and antagonizing the adverse effect of large amount steroid, which could inhibit the pulmonary fibrosis so as to affect the repairing of tissues.

There are many lethal factors that could cause death to SARS patients, among which secondary infection caused by serious destruction of immune function and utilization of steroid is an important one. In patients in the late stage, such infectionrelated syndromes as fungal infection, septicemia and disseminated intravascular coagulation could be seen. This study showed that there was a lowering trend of secondary infection of SARS in the treated group as compared with the control group, suggesting that Chinese drugs have certain effects in protecting cellular immunity and enhancing the resistance of the organism against infection.

Traditional Chinese medicine possesses lots of clinical experience and rational recognition on treatment of viral infected diseases, puts much thought on the principle of prevention first, and asserts that treatment of SARS should be done early and quick, with much stress paid to preventing its transferring. The recipes used in this study were formulated by several Chinese medical specialists who combined old and new experience. It could be prospected that the method and efficacy of ICWM treatment in treating SARS shall be further improved and bettered inevitably along with deepening research on the disease and precise screening on recipes and programs.

\section{REFERENCES}

1. Ministry of Health. Clinical Diagnostic Standard of Infectious Atypical Pneumonia. 2003.

(Received August 10, 2003) 